\title{
ON THE EXISTENCE OF GROUP INVERSES OF PEIRCE CORNER MATRICES
}

\author{
DAOCHANG ZHANG, DIJANA MOSIĆ, AND TIN-YAU TAM
}

\begin{abstract}
AвStRact. We give some statements that are equivalent to the existence of group inverses of Peirce corner matrices of a $2 \times 2$ block matrix and its generalized Schur complements. As applications, several new results for the Drazin inverses of the generalized Schur complements and the $2 \times 2$ block matrix are obtained and some of them generalize several results in the literature.
\end{abstract}

\section{INTRODUCTION}

The classical Sherman-Morrison-Woodbury formula

$$
\left(A-C D^{-1} B\right)^{-1}=A^{-1}+A^{-1} C\left(D-B A^{-1} C\right)^{-1} B A^{-1}
$$

expresses the inverse of the Schur complement $A-C D^{-1} B$ of $D$ of the $2 \times 2$ block matrix

$$
M=\left[\begin{array}{ll}
A & C \\
B & D
\end{array}\right]
$$

in terms of the inverses of $A$ and its Schur complement $D-B A^{-1} C$ of $M$, where $A$ and $D$ are invertible matrices, but not necessarily with the same size, and $B$ and $C$ are matrices with appropriate sizes such that $D-B A^{-1} C$ (and so $A-C D^{-1} B$ ) is invertible [23, 26]. There are applications of inverse matrix formulae of such type in various fields such as statistics, optimization, networks, numerical analysis, structural analysis, partial different equations [12]. Formulae of such type have been developed in the context of generalized inverses, such as the Moore-Penrose inverse [1, 19], the weighted Moore-Penrose inverse [24], the group inverse [6], the weighted Drazin inverse [9], the generalized Drazin inverse [10], and the Drazin inverse [27].

Researchers have tried to find formulae for the Drazin inverse of $M$ in terms of its blocks, where $A$ and $D$ are square matrices [13, 14, 20]. This

2000 Mathematics Subject Classification. 15A09; 15A30; 65F20.

Key words and phrases. Peirce corner matrix, generalized Schur complement, group inverse, Drazin inverse.

The first author is supported by the National Natural Science Foundation of China (NSFC) (No. 61672149; No. 51708091). The second author is supported by the Ministry of Education, Science and Technological Development, Republic of Serbia (No. 174007). 
problem originates from the singular system of differential equations [3, 5], and was proposed by Campbell and Meyer [4], but it is still an open problem if no additional assumptions are made on the blocks.

There are also some significant definitions in the ring theory. Let $R$ be a ring. A ring $S \subseteq R$ (with the same multiplication as $\mathrm{R}$, but not assumed to have an identity initially) is said to be a corner ring (or simply a corner) of $R$ if there exists an additive subgroup $C \subseteq R$ such that

$$
R=S \oplus C, \quad S \cdot C \subseteq C, \quad \text { and } \quad C \cdot S \subseteq C .
$$

In this case, we write $S<R$, and we call any subgroup $C$ satisfying (1.1) a complement of the corner ring $S$ in $R$. In general, such a complement $C$ is far from being unique. If a corner $S$ of a ring $R$ happens to have a unique complement, we shall call $S$ a rigid corner of $R$, and write $S \prec_{r} R$.

In 2006, Lam [17] proved that a corner ring of any ring $R$ must have an identity, although this may not be the identity of $R$.

Remark 1.1. [17, Proposition 2.2] Let $S<R$, with a complement $C$. If $1=e+f$ for some $e \in S$ and $f \in C$, then $e$ is an identity of the ring $S$. In particular, the decomposition $1=e+f$ is independent of the choice of the complement $C$, and $e, f$ are complementary idempotents in $R$.

Let $e, f$ be complementary idempotents in a ring $R$. Then $R_{e}$ is called the Peirce corner of $R$ (arising from the idempotent $e$ ) such that

(1) $R_{e}:=e R e<R$, which is the largest subring (resp. corner) of $R$ having $e$ as identity element.

(2) $R_{e} \prec_{r} R$ (i.e., $R_{e}$ is rigid in $R$ ), with a unique complement

$$
C_{e}:=f R e \oplus e R f \oplus f R f=\{r \in R: \text { ere }=0\},
$$

where $C_{e}$ is called the Peirce complement of $R_{e}$.

Recall that, for any ring $R$ (with identity), Jacobson's Lemma states that if $1-a b$ is invertible, then so is $1-b a$ and

$$
(1-b a)^{-1}=1+b(1-a b)^{-1} a \text {. }
$$

The group inverse of a complex square matrix $A$ is the unique matrix $A^{\#}$ such that

$$
A A^{\#} A=A, A^{\#} A A^{\#}=A^{\#}, A A^{\#}=A^{\#} A .
$$

The Drazin inverse of $A$ is the unique matrix $A^{d}$ such that

$$
A A^{d}=A^{d} A, A^{d} A A^{d}=A^{d}, A^{k}=A^{k+1} A^{d},
$$

where $k$ is the smallest non-negative integer such that $\operatorname{rank}\left(A^{k}\right)=\operatorname{rank}\left(A^{k+1}\right)$, called index of $A$ and denoted by ind $(A)$. We also denote $A^{e}=A A^{d}$ and $A^{\pi}=I-A^{e}$. If $\operatorname{ind}(A)=1$, then $A^{d}=A^{\#}$. 
In this paper, our aim is, by the Peirce corner theory, to establish a relationship between group inverses of a $2 \times 2$ block matrix and its generalized Schur complements, and to find statements that are equivalent to the existence of group inverses of Peirce corner matrices of a $2 \times 2$ block matrix and its generalized Schur complements. Utilizing the equivalent statements, we derive some new results about Drazin inverses of the generalized Schur complements and the $2 \times 2$ block matrix, and generalize several results in the literature.

Throughout this paper, for notational convenience, we denote

$$
\begin{gathered}
S=A-C D^{d} B, \quad s=A^{e} S A^{e}, \quad Z=D-B A^{d} C, \quad z=D^{e} Z D^{e}, \\
M=\left[\begin{array}{ll}
A & C \\
B & D
\end{array}\right], \quad E=\left[\begin{array}{cc}
A^{e} & 0 \\
0 & D^{e}
\end{array}\right], \quad M_{E}=E M E=\left[\begin{array}{cc}
A A^{e} & A^{e} C D^{e} \\
D^{e} B A^{e} & D D^{e}
\end{array}\right],
\end{gathered}
$$

where $A \in \mathbb{C}^{n \times n}, B \in \mathbb{C}^{m \times n}, C \in \mathbb{C}^{n \times m}$, and $D \in \mathbb{C}^{m \times m}$. Here $\mathbb{C}^{m \times n}$ is the set of $m \times n$ complex matrices. We treat $\sum_{i=m}^{n} *=0$ whenever $m<n$, which is used in Section 3 and Section 4 . We denote by $I$ the identity matrix of proper size.

\section{EXISTENCES OF GROUP INVERSES}

In this section, we give statements that are equivalent to the existence of group inverses of Peirce corner matrices of a $2 \times 2$ block matrix and its generalized Schur complements.

Here we cite a result which characterizes matrices with the same eigenprojection.

Lemma 2.1. [7, Theorem 2.1] Let $A, B \in \mathbb{C}^{n \times n}$ be Drazin invertible. Then the following conditions are equivalent:

(1) $A^{\pi}=B^{\pi}$;

(2) $A^{\pi} B=B A^{\pi}, B A^{\pi}$ is nilpotent and $B+A^{\pi}$ is nonsingular;

(3) $I+A^{d}(B-A)$ is nonsingular, $A^{\pi} B=B A^{\pi}$ and $B A^{\pi}$ is nilpotent;

(4) $B^{d}=\left(I+A^{d}(B-A)\right)^{-1} A^{d}$;

(5) $B^{d}-A^{d}=A^{d}(A-B) B^{d}$.

Now we can give our first main result.

Theorem 2.2. The following statements are equivalent:

(1) $M_{E}^{\#}$ exists, and $M_{E}^{e}=E$;

(2) $s^{\#}$ exists, and $s^{e}=A^{e}$;

(3) $z^{\#}$ exists, and $z^{e}=D^{e}$;

(4) $A^{\pi}+s$ is invertible;

(5) $I+A^{d}(s-A)$ is invertible $\left(\right.$ or $I+A^{d}(S-I) A$ is invertible $)$;

(6) $s^{d}=\left(I+A^{d}(s-A)\right)^{-1} A^{d}$;

(7) $D^{\pi}+z$ is invertible; 
(8) $I+D^{d}(z-D)$ is invertible $\left(\right.$ or $I+D^{d}(Z-I) D$ is invertible);

(9) $z^{d}=\left(I+D^{d}(z-D)\right)^{-1} D^{d}$;

(10) $s^{\#}, z^{\#}$ exist, $s^{\#}=A^{d} C z^{\#} B A^{d}+A^{d}$, and $A^{d} C z^{\#}=s^{\#} C D^{d}$;

(11) $s^{\#}, z^{\#}$ exist, $z^{\#}=D^{d} B s^{\#} C D^{d}+D^{d}$, and $D^{d} B s^{\#}=z^{\#} B A^{d}$;

(12) $M_{E}^{\#}$ exists, and

$$
M_{E}^{\#}=\left[\begin{array}{cc}
A^{d}+A^{d} C z^{\#} B A^{d} & -A^{d} C z^{\#} \\
-z^{\#} B A^{d} & z^{\#}
\end{array}\right]=\left[\begin{array}{cc}
s^{\#} & -s^{\#} C D^{d} \\
-D^{d} B s^{\#} & D^{d} B s^{\#} C D^{d}+D^{d}
\end{array}\right] .
$$

Proof. We first show the implication $(1) \Rightarrow(3)$ holds. Note that we have the following two representations for the Peirce corner matrix $M_{E}$ of $M$.

$$
\left[\begin{array}{cc}
A A^{e} & A^{e} C D^{e} \\
D^{e} B A^{e} & D D^{e}
\end{array}\right]=\left[\begin{array}{cc}
A^{e} & 0 \\
D^{e} B A^{d} & D^{e}
\end{array}\right]\left[\begin{array}{cc}
A A^{e} & 0 \\
0 & D D^{e}-D^{e} B A^{d} C D^{e}
\end{array}\right]\left[\begin{array}{cc}
A^{e} & A^{d} C D^{e} \\
0 & D^{e}
\end{array}\right],
$$

$$
\left[\begin{array}{cc}
A A^{e} & A^{e} C D^{e} \\
D^{e} B A^{e} & D D^{e}
\end{array}\right]=\left[\begin{array}{cc}
A^{e} & A^{e} C D^{d} \\
0 & D^{e}
\end{array}\right]\left[\begin{array}{cc}
A A^{e}-A^{e} C D^{d} B A^{e} & 0 \\
0 & D D^{e}
\end{array}\right]\left[\begin{array}{cc}
A^{e} & 0 \\
D^{d} B A^{e} & D^{e}
\end{array}\right] .
$$

Let $\mathbb{W}=\left\{M \in \mathbb{C}^{n \times n}\right\}$ and $\mathbb{W}_{E}=\left\{M_{E} \in \mathbb{C}^{n \times n}\right\}$. We observe that all of the matrices above are contained in the Peirce corner $\mathbb{W}_{E}$ of $\mathbb{W}$ with identity $E$. Since $\mathbb{W}_{E}$ is a finite dimensional algebra over $\mathbb{C}$ with identity $E$, by $E M_{E}=M_{E} E=M_{E}$, we conclude that $\mathbb{W}_{E}$ is Dedekind finite (see [18, Corollary 21.27]), i.e., if $X Y=E$ then $Y X=E$ for the arbitrary $X, Y \in \mathbb{W}_{E}$.

Since

$$
\left[\begin{array}{cc}
A^{e} & 0 \\
-D^{e} B A^{d} & D^{e}
\end{array}\right] M_{E}\left[\begin{array}{cc}
A^{e} & -A^{d} C D^{e} \\
0 & D^{e}
\end{array}\right]=\left[\begin{array}{cc}
A A^{e} & 0 \\
0 & D^{e} Z D^{e}
\end{array}\right]
$$

and

$$
\left[\begin{array}{cc}
A A^{e} & 0 \\
0 & D^{e} Z D^{e}
\end{array}\right] \times\left[\begin{array}{cc}
A^{e} & A^{d} C D^{e} \\
0 & D^{e}
\end{array}\right] M_{E}^{\#}\left[\begin{array}{cc}
A^{e} & 0 \\
D^{e} B A^{d} & D^{e}
\end{array}\right]=E
$$

we conclude that $\left[\begin{array}{cc}A A^{e} & 0 \\ 0 & D^{e} Z D^{e}\end{array}\right]^{\#}$ exists, and $\left[\begin{array}{cc}A A^{e} & 0 \\ 0 & D^{e} Z D^{e}\end{array}\right]^{e}=E$. Then, by the uniqueness of the group inverse, $z^{\#}$ exists and $z^{e}=D^{e}$.

Similarly, it can be proved that (1), (2), and (3) are equivalent.

To prove that the implications $(1) \Rightarrow(10)$ and $(1) \Rightarrow(11)$ hold, we combine the equivalence of (1), (2), and (3) to give

$$
\begin{aligned}
& {\left[\begin{array}{cc}
A^{e} & -A^{d} C D^{e} \\
0 & D^{e}
\end{array}\right]\left[\begin{array}{cc}
A^{d} & 0 \\
0 & z^{\#}
\end{array}\right]\left[\begin{array}{cc}
A^{e} & 0 \\
-D^{e} B A^{d} & D^{e}
\end{array}\right] } \\
= & {\left[\begin{array}{cc}
A^{e} & 0 \\
-D^{d} B A^{e} & D^{e}
\end{array}\right]\left[\begin{array}{cc}
s^{\#} & 0 \\
0 & D^{d}
\end{array}\right]\left[\begin{array}{cc}
A^{e} & -A^{e} C D^{d} \\
0 & D^{e}
\end{array}\right], }
\end{aligned}
$$


i.e.,

$$
\left[\begin{array}{cc}
A^{d}+A^{d} C z^{\#} B A^{d} & -A^{d} C z^{\#} \\
-z^{\#} B A^{d} & z^{\#}
\end{array}\right]=\left[\begin{array}{cc}
s^{\#} & -s^{\#} C D^{d} \\
-D^{d} B s^{\#} & D^{d} B s^{\#} C D^{d}+D^{d}
\end{array}\right],
$$

and so

$$
\begin{aligned}
& s^{\#}=A^{d}+A^{d} C z^{\#} B A^{d}, \quad s^{\#} C D^{d}=A^{d} C z^{\#}, \\
& D^{d} B s^{\#}=z^{\#} B A^{d}, \quad z^{\#}=D^{d} B s^{\#} C D^{d}+D^{d} .
\end{aligned}
$$

The implication $(10) \Rightarrow(2)$ holds because

$s^{\#}=A^{d} C z^{\#} B A^{d}+A^{d}=s^{\#} C D^{d} B A^{d}+A^{d}=s^{\#}(A-S) A^{d}+A^{d}=s^{\#}-s^{\#} S A^{d}+A^{d}$ implies $A^{d}=s^{\#} S A^{d}$. Hence, $A^{e}=A^{d} A=s^{\#} S A^{d} A=s^{\#} A^{e} S A^{e}=s^{\#} s=s^{e}$.

In a similar way, we can verify that $(11) \Rightarrow(3)$. Thus, (1), (2), (3), (10), and (11) are equivalent.

By (2.1), (2.2), and simple calculation, we obtain the representation of $M_{E}^{\#}$ in (12) and the equivalence between (12), and (1)-(3) holds.

The rest can be obtained by Lemma2.1 and Jacobson's Lemma.

The equivalent statements in the above theorem distinctly provide relationship between the existence of group inverses of Peirce corner matrices of a $2 \times 2$ block matrix and its generalized Schur complements. Although some extra assumptions are imposed, the theorem yields a new method in the research field and can combine the two different research directions of the generalized inverses of the generalized Schur complements and the $2 \times 2$ block matrix. The result is applied to Section 3 and Section 4, and generalizes a few results in the literature.

We next conclude with a remark to provide for the application in Section 3.

Remark 2.3. We note that [27, Lemma 2.4] states if $A^{d} C D^{\pi} Z^{d} B A^{d}=$ $A^{d} C D^{d} Z^{\pi} B A^{d}$, then $s^{\#}$ exists and $s^{\#}=A^{d}+A^{d} C Z^{d} B A^{d}$, and so $s^{e}=A^{e}$. In this case, by Theorem 2.2, $s^{\#}=A^{d}+A^{d} C z^{\#} B A^{d}$. Since the group inverse of $s$ is unique, we can obtain if $A^{d} C D^{\pi} Z^{d} B A^{d}=A^{d} C D^{d} Z^{\pi} B A^{d}$, then

$$
A^{d} C Z^{d} B A^{d}=A^{d} C z^{\#} B A^{d} .
$$

We can also conclude that if $D^{d} B A^{\pi} S^{d} C D^{d}=D^{d} B A^{d} S^{\pi} C D^{d}$, then $z^{\#}$ exists, $z^{\#}=D^{d}+D^{d} B S^{d} C D^{d}$ and $z^{e}=D^{e}$. Similarly, if $D^{d} B A^{\pi} S^{d} C D^{d}=$ $D^{d} B A^{d} S^{\pi} C D^{d}$, then

$$
D^{d} B S^{d} C D^{d}=D^{d} B s^{\#} C D^{d} .
$$

Applying Jacobson's Lemma, we can obtain equivalent statements that are similar to those in Theorem 2.2, Furthermore, similar to Theorem 2.2, by [16, Theorem 2.3] or [7, Lemma 1.1], we can verify a remark as follows. 
Remark 2.4. (a) Let $Z=D-B A^{d} C, z_{1}=D^{\pi} Z D^{\pi}$,

$$
M=\left[\begin{array}{ll}
A & C \\
B & D
\end{array}\right], G=\left[\begin{array}{cc}
A^{e} & 0 \\
0 & D^{\pi}
\end{array}\right], M_{G}=G M G=\left[\begin{array}{cc}
A A^{e} & A^{e} C D^{\pi} \\
D^{\pi} B A^{e} & D D^{\pi}
\end{array}\right],
$$

where $A \in \mathbb{C}^{n \times n}, B \in \mathbb{C}^{m \times n}, C \in \mathbb{C}^{n \times m}$, and $D \in \mathbb{C}^{m \times m}$. The following statements are equivalent:

(1) $M_{G}^{\#}$ exists, and $M_{G}^{e}=G$;

(2) $z_{1}^{\#}$ exists, and $z_{1}^{e}=D^{\pi}$;

(3) $D^{e}+z_{1}$ is invertible;

(4) $I+D^{\pi}\left(z_{1}-I\right)$ is invertible (or $I+D^{\pi}(Z-I)$ is invertible);

(5) $M_{G}^{\#}$ exists, and

$$
M_{G}^{\#}=\left[\begin{array}{cc}
A^{d}+A^{d} C z_{1}^{\#} B A^{d} & -A^{d} C z_{1}^{\#} \\
-z_{1}^{\#} B A^{d} & z_{1}^{\#}
\end{array}\right] .
$$

(b) Let $S=A-C D^{d} B, s_{1}=A^{\pi} S A^{\pi}$,

$$
M=\left[\begin{array}{ll}
A & C \\
B & D
\end{array}\right], H=\left[\begin{array}{cc}
A^{\pi} & 0 \\
0 & D^{e}
\end{array}\right], M_{H}=H M H=\left[\begin{array}{cc}
A A^{\pi} & A^{\pi} C D^{e} \\
D^{e} B A^{\pi} & D D^{e}
\end{array}\right],
$$

where $A \in \mathbb{C}^{n \times n}, B \in \mathbb{C}^{m \times n}, C \in \mathbb{C}^{n \times m}, D \in \mathbb{C}^{m \times m}$. The following statements are equivalent:

(1) $M_{H}^{\#}$ exists, and $M_{H}^{e}=H$;

(2) $s_{1}^{\#}$ exists, and $s_{1}^{e}=A^{\pi}$;

(3) $A^{e}+s_{1}$ is invertible;

(4) $I+A^{\pi}\left(s_{1}-I\right)$ is invertible (or $I+A^{\pi}(S-I)$ is invertible);

(5) $M_{H}^{\#}$ exists, and

$$
M_{H}^{\#}=\left[\begin{array}{cc}
s_{1}^{\#} & -s_{1}^{\#} C D^{d} \\
-D^{d} B s_{1}^{\#} & D^{d} B s_{1}^{\#} C D^{d}+D^{d}
\end{array}\right] .
$$

By direct calculation, we can obtain the following corollary.

Corollary 2.5. (a) The following statements are equivalent:

(1) $M_{E}^{\#}$ exists, and

$$
M_{E}^{\#}=\left[\begin{array}{cc}
A^{d}+A^{d} C z^{d} B A^{d} & -A^{d} C z^{d} \\
-z^{d} B A^{d} & z^{d}
\end{array}\right]
$$

(2) $z^{\#}$ exists, $A^{d} C z^{\pi} D^{e}=0$ and $D^{e} z^{\pi} B A^{d}=0$;

(3) $z^{\#}$ exists, $A^{d} C z^{e}=A^{d} C D^{e}$ and $D^{e} B A^{d}=z^{e} B A^{d}$.

(b) The following statements are equivalent:

(1) $M_{E}^{\#}$ exists, and

$$
M_{E}^{\#}=\left[\begin{array}{cc}
s^{d} & -s^{d} C D^{d} \\
-D^{d} B s^{d} & D^{d} B s^{d} C D^{d}+D^{d}
\end{array}\right]
$$


(2) $s^{\#}$ exists, $A^{e} s^{\pi} C D^{d}=0$ and $D^{d} B s^{\pi} A^{e}=0$;

(3) $s^{\#}$ exists, $A^{e} C D^{d}=s^{e} C D^{d}$ and $D^{d} B s^{e}=D^{d} B A^{e}$.

We note that $M_{E}^{e}=\left[\begin{array}{cc}A^{e} & 0 \\ 0 & z^{e}\end{array}\right]$ in Corollary 2.5)(a). If we assume $A^{d} C z^{e}=$ $A^{d} C D^{e}$ and $D^{e} B A^{d}=z^{e} B A^{d}$, then $A^{d} C D^{\pi} z^{d} B A^{d}=0=A^{d} C D^{d} z^{\pi} B A^{d}$.

In the following result, we present necessary and sufficient conditions for the solvability of a matrix equation, and give the the expression of the general solution if the system is solvable.

Theorem 2.6. Let $A \in \mathbb{C}^{n \times n}, B \in \mathbb{C}^{m \times n}, C \in \mathbb{C}^{n \times m}$, and $D \in \mathbb{C}^{m \times m}$. The system

$$
\left[\begin{array}{ll}
A & C \\
B & D
\end{array}\right]\left[\begin{array}{l}
A^{e} X \\
Z^{e} Y
\end{array}\right]=\left[\begin{array}{c}
A^{e} \\
0
\end{array}\right]
$$

has a solution if and only if $A^{\pi} C Z^{d} B A^{d}=0$ and $Z^{\pi} B A^{d}=0$. In this case, the general solution is

$$
X=A^{d}+A^{d} C Z^{d} B A^{d}+A^{\pi} U \text { and } Y=-Z^{d} B A^{d}+Z^{\pi} V,
$$

for arbitrary $U \in \mathbb{C}^{n \times n}$ and $V \in \mathbb{C}^{m \times n}$.

Proof. If the system (2.3) has a solution, then

$$
A A^{e} X+C Z^{e} Y=A^{e},
$$

and

$$
B A^{e} X+D Z^{e} Y=0
$$

By (2.4), we have

$$
A^{e} X=A^{d}-A^{d} C Z^{e} Y
$$

which is substituted into (2.5) to have $B A^{d}-B A^{d} C Z^{e} Y+D Z^{e} Y=0$, i.e.,

$$
-B A^{d}=\left(D-B A^{d} C\right) Z^{e} Y=Z Z^{e} Y \text {. }
$$

Then

$$
Z^{e} Y=-Z^{d} B A^{d}
$$

which is substituted into (2.6) to have

$$
A^{e} X=A^{d}+A^{d} C Z^{d} B A^{d} .
$$

Therefore,

$$
\begin{aligned}
A^{e} & =A\left(A^{e} X\right)+C\left(Z^{e} Y\right)=A A^{d}+A A^{d} C Z^{d} B A^{d}-C Z^{d} B A^{d} \\
& =A^{e}-A^{\pi} C Z^{d} B A^{d}
\end{aligned}
$$


gives $A^{\pi} C Z^{d} B A^{d}=0$. We obtain

$$
\begin{aligned}
0 & =B\left(A^{e} X\right)+D\left(Z^{e} Y\right)=B A^{d}+B A^{d} C Z^{d} B A^{d}-D Z^{d} B A^{d} \\
& =B A^{d}-Z Z^{d} B A^{d}=Z^{\pi} B A^{d} .
\end{aligned}
$$

Following [2, p. 52], we get the general solution of $A^{e} X=A^{d}+A^{d} C Z^{d} B A^{d}$ as $X=A^{d}+A^{d} C Z^{d} B A^{d}+A^{\pi} U$, for arbitrary $U \in \mathbb{C}^{n \times n}$. Similarly, the general solution of the matrix equation $Z^{e} Y=-Z^{d} B A^{d}$ is $Y=-Z^{d} B A^{d}+Z^{\pi} V$, for arbitrary $V \in \mathbb{C}^{m \times n}$.

Suppose that $A^{\pi} C Z^{d} B A^{d}=0$ and $Z^{\pi} B A^{d}=0$. Set $X=A^{d}+A^{d} C Z^{d} B A^{d}+$ $A^{\pi} U$ and $Y=-Z^{d} B A^{d}+Z^{\pi} V$, for arbitrary $U \in \mathbb{C}^{n \times n}$ and $V \in \mathbb{C}^{m \times n}$. We can easily verify that $A A^{e} X+C Z^{e} Y=A^{e}$ and $B A^{e} X+D Z^{e} Y=0$, i.e., the system (2.3) has a solution.

We next consider the group inverse of $s$ under the above equivalence restriction.

Corollary 2.7. Let $A \in \mathbb{C}^{n \times n}, B \in \mathbb{C}^{m \times n}, C \in \mathbb{C}^{n \times m}, D \in \mathbb{C}^{m \times m}$, and let $A^{d} C D^{\pi} Z^{d} B A^{d}=0$. If the system (2.3) has a solution, then $s^{\#}$ exists,

$$
s^{\#}=A^{d}+A^{d} C Z^{d} B A^{d} \text {, }
$$

and $s^{e}=A^{e}$.

Proof. Note that (2.4) $-C D^{d} \times(2.5)$ yields

$$
A^{e}=A A^{e} X+C Z^{e} Y-C D^{d} B A^{e} X-C D^{e} Z^{e} Y .
$$

Then

$$
A^{e}=A A^{e} X-A^{e} C D^{d} B A^{e} X+A^{e} C D^{\pi} Z^{e} Y,
$$

which implies, by (2.7) and $A^{d} C D^{\pi} Z^{d} B A^{d}=0$,

$$
A^{e}=A A^{e} X-A^{e} C D^{d} B A^{e} X=s A^{e} X .
$$

By (2.8),

$$
A^{e}=s\left(A^{d}+A^{d} C Z^{d} B A^{d}\right) .
$$

Since $\mathbb{W}_{A^{e}}=\left\{A^{e} M^{\prime} A^{e} \in \mathbb{C}^{n \times n}: M^{\prime} \in \mathbb{C}^{n \times n}\right\}$ is a finite dimensional algebra over $\mathbb{C}$ with identity $A^{e}$, by $A^{e} M_{A^{e}}=M_{A^{e}} A^{e}=M_{A^{e}}$, we conclude that $\mathbb{W}_{A^{e}}$ is also Dedekind finite as in the proof of Theorem 2.2. Then

$$
A^{e}=\left(A^{d}+A^{d} C Z^{d} B A^{d}\right) s .
$$

Thus $s^{\#}$ exists,

$$
s^{\#}=A^{d}+A^{d} C Z^{d} B A^{d},
$$

and $s^{e}=A^{e}$. 
3. APPLICATIONS to $S^{d}$ AND $Z^{d}$

Applying Theorem 2.2, we obtain new results for the Drazin inverses of the generalized Schur complements $S$ and $Z$ in this section. As a consequence, we generalize several results in the literature, and recover some generalized Sherman-Morrison-Woodbury formulae.

We first need a existing lemma. In what follows, recall that $s=A A^{d} S A A^{d}$ and $z=D D^{d} Z D D^{d}$.

Lemma 3.1. [27] If $A^{\pi} C D^{d} B=0$, then $s=S A A^{d}$ and

$$
S^{d}=s^{d}+\sum_{i=0}^{k-1}\left(s^{d}\right)^{i+2} S A^{i} A^{\pi}
$$

where $k=\operatorname{ind}(A)$.

By Lemma 3.1 and Theorem 2.2, we get the following result.

Theorem 3.2. If any of (1)-(12) in Theorem 2.2 is valid, and $A^{\pi} C D^{d} B=0$, then

$$
S^{d}=s^{\#}+\sum_{i=0}^{k-1}\left(s^{\#}\right)^{i+2} S A^{i} A^{\pi},
$$

where $s^{\#}=A^{d} C z^{\#} B A^{d}+A^{d}$, and $k=\operatorname{ind}(A)$.

The following conclusion follows from Remark 2.3 and Theorem 3.2 , because $A^{d} C D^{\pi} Z^{d} B A^{d}=A^{d} C D^{d} Z^{\pi} B A^{d}$ implies that $s^{\#}$ exists and $s^{e}=A^{e}$, which belongs in one of the equivalent statements in Theorem 2.2, and also $A^{d} C Z^{d} B A^{d}=A^{d} C z^{\#} B A^{d}$.

Corollary 3.3. [27, Theorem 2.5] If $A^{\pi} C D^{d} B=0$ and $A^{d} C D^{\pi} Z^{d} B A^{d}=$ $A^{d} C D^{d} Z^{\pi} B A^{d}$, then

$$
S^{d}=A^{d}+A^{d} C Z^{d} B A^{d}+\sum_{i=0}^{k-1}\left(A^{d}+A^{d} C Z^{d} B A^{d}\right)^{i+2} S A^{i} A^{\pi},
$$

or alternatively

$$
\begin{aligned}
S^{d}=A^{d}+A^{d} C Z^{d} B A^{d} & -\sum_{i=0}^{k-1}\left(A^{d}+A^{d} C Z^{d} B A^{d}\right)^{i+1} A^{d} C Z^{d} B A^{i} A^{\pi} \\
& +\sum_{i=0}^{k-1}\left(A^{d}+A^{d} C Z^{d} B A^{d}\right)^{i+1} A^{d} C\left(Z^{d} D^{\pi}-Z^{\pi} D^{d}\right) B A^{i},
\end{aligned}
$$

where $k=\operatorname{ind}(A)$. 
We can see from [27] how Corollary 3.3 gives and generalizes the ShermanMorrison-Woodbury formula and some results in [11, 21, 22, 25].

The following corollary, which is a dual version of Theorem 3.2, can be proved similarly.

Corollary 3.4. If any of (1)-(12) in Theorem 2.2 is valid, and $C D^{d} B A^{\pi}=0$, then $s=A A^{d} S$, and

$$
S^{d}=s^{\#}+\sum_{i=0}^{k-1} A^{\pi} A^{i} S\left(s^{\#}\right)^{i+2}
$$

where $s^{\#}=A^{d} C z^{\#} B A^{d}+A^{d}$, and $k=\operatorname{ind}(A)$.

We would like to point out that Corollary 3.4 generalizes [21, Theorem 3], [11, Theorem 2.2], [22, Theorem 2.2], and [25, Theorem 2.1].

Similarly, we can obtain the following result.

Corollary 3.5. If any of (1)-(12) in Theorem 2.2 is valid, and $D^{\pi} B A^{d} C=0$, then $z=Z D D^{d}$ and

$$
Z^{d}=z^{\#}+\sum_{i=0}^{k-1}\left(z^{\#}\right)^{i+2} Z D^{i} D^{\pi}
$$

where $z^{\#}=D^{d} B s^{\#} C D^{d}+D^{d}$, and $k=\operatorname{ind}(D)$.

\section{APPLICATIONS TO $M^{d}$}

Under new conditons, using Theorem 2.2, we present several representations for the Drazin inverse of a $2 \times 2$ block matrix $M$ in terms of its blocks and also elements $z$ and $s$ which include corresponding generalized Schur complements.

We cite one result as follows.

Lemma 4.1. [15, Theorem 2.1] Let $P$ and $Q \in \mathbb{C}^{n \times n}$. If $P Q=0$, then

$$
(P+Q)^{d}=Q^{\pi} \sum_{i=0}^{t-1} Q^{i}\left(P^{d}\right)^{i+1}+\sum_{i=0}^{s-1}\left(Q^{d}\right)^{i+1} P^{i} P^{\pi},
$$

where $s=\operatorname{ind}(P)$ and $t=\operatorname{ind}(Q)$.

In the case that one of equivalent statements of Theorem 2.2 holds, $A C D^{\pi}=$ 0 and $D B A^{\pi}=0$, we obtain the following representation for the Drazin inverse of $M$. 
Theorem 4.2. If any of (1)-(12) of Theorem 2.2 is valid, $A C D^{\pi}=0$, and $D B A^{\pi}=0$, then

$$
\begin{aligned}
& M^{d}=\left(\left[\begin{array}{cc}
\left(C B A^{\pi}\right)^{\pi} & 0 \\
0 & \left(B C D^{\pi}\right)^{\pi}
\end{array}\right]\right. \\
& \text { - } \left.\sum_{m=1}^{t-1}\left[\begin{array}{cc}
0 & \left(C B A^{\pi}\right)^{d} C D^{\pi} \\
B A^{\pi}\left(C B A^{\pi}\right)^{d} & 0
\end{array}\right]^{m}\left[\begin{array}{cc}
A^{m} A^{\pi} & 0 \\
0 & D^{m} D^{\pi}
\end{array}\right]\right) \\
& \times \sum_{i=0}^{r-1}\left[\begin{array}{ll}
A A^{\pi} & C D^{\pi} \\
B A^{\pi} & D D^{\pi}
\end{array}\right]^{i}\left(I+\left[\begin{array}{cc}
0 & A^{\pi} C D^{e} \\
D^{\pi} B A^{e} & 0
\end{array}\right] P_{1}^{\#}\right)\left(P_{1}^{\#}\right)^{i+1} \\
& +\left(\left[\begin{array}{cc}
0 & \left(C B A^{\pi}\right)^{d} C D^{\pi} \\
B A^{\pi}\left(C B A^{\pi}\right)^{d} & 0
\end{array}\right]\right. \\
& \left.+\sum_{n=1}^{t-1}\left[\begin{array}{cc}
0 & \left(C B A^{\pi}\right)^{d} C D^{\pi}\left(C B A^{\pi}\right)^{d} \\
0
\end{array}\right]^{n+1}\left[\begin{array}{cc}
A^{n} A^{\pi} & 0 \\
0 & D^{n} D^{\pi}
\end{array}\right]\right) \\
& \times\left(\left[\begin{array}{cc}
A^{\pi} & 0 \\
0 & D^{\pi}
\end{array}\right]-\left[\begin{array}{cc}
0 & A^{\pi} C D^{e} \\
D^{\pi} B A^{e} & 0
\end{array}\right] P_{1}^{\#}\right)
\end{aligned}
$$

where $t=\max \{\operatorname{ind}(A), \operatorname{ind}(D)\}, r=\operatorname{ind}\left(\left[\begin{array}{cc}A A^{\pi} & C D^{\pi} \\ B A^{\pi} & D D^{\pi}\end{array}\right]\right)$, and

$$
P_{1}^{\#}=\left[\begin{array}{cc}
A^{d}+A^{d} C z^{\#} B A^{d} & -A^{d} C z^{\#} \\
-z^{\#} B A^{d} & z^{\#}
\end{array}\right]=\left[\begin{array}{cc}
s^{\#} & -s^{\#} C D^{d} \\
-D^{d} B s^{\#} & D^{d} B s^{\#} C D^{d}+D^{d}
\end{array}\right] .
$$

Proof. We can write $M=P+Q$, where

$$
P=\left[\begin{array}{ll}
A A^{e} & C D^{e} \\
B A^{e} & D D^{e}
\end{array}\right] \text { and } Q=\left[\begin{array}{ll}
A A^{\pi} & C D^{\pi} \\
B A^{\pi} & D D^{\pi}
\end{array}\right]
$$

Since $P Q=0$, then, by Lemma 4.1,

$$
M^{d}=Q^{\pi} \sum_{i=0}^{\operatorname{ind}(Q)-1} Q^{i}\left(P^{d}\right)^{i+1}+\sum_{i=0}^{\operatorname{ind}(P)-1}\left(Q^{d}\right)^{i+1} P^{i} P^{\pi} .
$$

Suppose that $P=P_{1}+P_{2}$, where

$$
P_{1}=\left[\begin{array}{cc}
A A^{e} & A^{e} C D^{e} \\
D^{e} B A^{e} & D D^{e}
\end{array}\right] \text { and } P_{2}=\left[\begin{array}{cc}
0 & A^{\pi} C D^{e} \\
D^{\pi} B A^{e} & 0
\end{array}\right]
$$

Then $P_{1} P_{2}=0$ and $P_{2}^{2}=0$. Using Lemma 4.1, we get

$$
P^{d}=P_{1}^{\#}+P_{2}\left(P_{1}^{\#}\right)^{2}=\left(I+P_{2} P_{1}^{\#}\right) P_{1}^{\#},
$$


where the expression for $P_{1}^{\#}$ follows by Theorem 2.2. By $P_{1} P_{1}^{\#}=\left[\begin{array}{cc}A^{e} & 0 \\ 0 & D^{e}\end{array}\right]$, we observe that, for $i=1,2, \ldots$,

$$
P^{i} P^{\pi}=\left[\begin{array}{cc}
A A^{e} & C D^{e} \\
B A^{e} & D D^{e}
\end{array}\right]^{i}\left(\left[\begin{array}{cc}
A^{\pi} & 0 \\
0 & D^{\pi}
\end{array}\right]-\left[\begin{array}{cc}
0 & A^{\pi} C D^{e} \\
D^{\pi} B A^{e} & 0
\end{array}\right] P_{1}^{\#}\right)=0
$$

For

$$
Q_{1}=\left[\begin{array}{cc}
A A^{\pi} & 0 \\
0 & D D^{\pi}
\end{array}\right] \text { and } Q_{2}=\left[\begin{array}{cc}
0 & C D^{\pi} \\
B A^{\pi} & 0
\end{array}\right],
$$

we have $Q=Q_{1}+Q_{2}, Q_{1} Q_{2}=0$ and $Q_{1}$ is nilpotent. By [8, Theorem 2.1],

$$
Q_{2}^{d}=\left[\begin{array}{cc}
0 & \left(C B A^{\pi}\right)^{d} C D^{\pi} \\
B A^{\pi}\left(C B A^{\pi}\right)^{d} & 0
\end{array}\right] .
$$

Applying Lemma 4.1 again, we obtain

$$
Q^{d}=\sum_{n=0}^{\operatorname{ind}\left(Q_{1}\right)-1}\left(Q_{2}^{d}\right)^{n+1} Q_{1}^{n}
$$

and so

$$
Q^{\pi}=Q_{2}^{\pi}-\sum_{m=1}^{\operatorname{ind}\left(Q_{1}\right)-1}\left(Q_{2}^{d}\right)^{m} Q_{1}^{m}
$$

By direct computation, we obtain (4.1).

As a consequence of Theorem 4.2, we obtain another expressions for $M^{d}$.

Corollary 4.3. Let $t, r$ and $P_{1}^{\#}$ be defined as in Theorem 4.2

(a) If $A C=0$ and $D B=0$, then

$$
\begin{aligned}
M^{d} & =\left(\left[\begin{array}{cc}
\left(C B A^{\pi}\right)^{\pi} & 0 \\
0 & \left(B C D^{\pi}\right)^{\pi}
\end{array}\right]\right. \\
& \left.-\sum_{m=1}^{t-1}\left[\begin{array}{cc}
0 & \left(C B A^{\pi}\right)^{d} C D^{\pi} \\
B\left(C B A^{\pi}\right)^{d} & 0
\end{array}\right]^{m}\left[\begin{array}{cc}
A^{m} A^{\pi} & 0 \\
0 & D^{m} D^{\pi}
\end{array}\right]\right) \\
& \times \sum_{i=0}^{r-1}\left[\begin{array}{cc}
A A^{\pi} & C D^{\pi} \\
B A^{\pi} & D D^{\pi}
\end{array}\right]^{i}\left[\begin{array}{cc}
\left(A^{d}\right)^{i+1} & C\left(D^{d}\right)^{i+2} \\
B\left(A^{d}\right)^{i+2} & \left(D^{d}\right)^{i+1}
\end{array}\right] \\
& +\left[\begin{array}{cc}
-\left(C B A^{\pi}\right)^{d} C B A^{d} & \left(C B A^{\pi}\right)^{d} C D^{\pi} \\
B\left(C B A^{\pi}\right)^{d} & -B\left(C B A^{\pi}\right)^{d} C D^{d}
\end{array}\right] \\
& +\sum_{n=1}^{t-1}\left[\begin{array}{cc}
0 & \left(C B A^{\pi}\right)^{d} C D^{\pi} \\
B\left(C B A^{\pi}\right)^{d} & 0
\end{array}\right]^{n+1}\left[\begin{array}{cc}
A^{n} A^{\pi} & 0 \\
0 & D^{n} D^{\pi}
\end{array}\right] .
\end{aligned}
$$


(b) If any of (1)-(12) in Theorem 2.2 is valid, $C D^{\pi}=0$ and $B A^{\pi}=0$, then

$$
M^{d}=\sum_{i=0}^{t-1}\left[\begin{array}{cc}
A A^{\pi} & 0 \\
0 & D D^{\pi}
\end{array}\right]^{i}\left(I+\left[\begin{array}{cc}
0 & A^{\pi} C \\
D^{\pi} B & 0
\end{array}\right] P_{1}^{\#}\right)\left(P_{1}^{\#}\right)^{i+1} .
$$

If any one of equivalent statements in Theorem 2.2 holds, $A^{\pi} C D=0$, and $D^{\pi} B A=0$, then one more formula for $M^{d}$ is given.

Theorem 4.4. If any one of (1)-(12) in Theorem 2.2 is valid, $A^{\pi} C D=0$, and $D^{\pi} B A=0$, then

$$
\begin{aligned}
& M^{d}=\left(\left[\begin{array}{cc}
A^{\pi} & 0 \\
0 & D^{\pi}
\end{array}\right]-P_{1}^{\#}\left[\begin{array}{cc}
0 & A^{e} C D^{\pi} \\
D^{e} B A^{\pi} & 0
\end{array}\right]\right) \\
& \times\left[\begin{array}{cc}
0 & \left(A^{\pi} C B\right)^{d} A^{\pi} C \\
D^{\pi} B\left(A^{\pi} C B\right)^{d} & 0
\end{array}\right] \\
& \left.+\sum_{n=1}^{t-1}\left[\begin{array}{cc}
A^{n} A^{\pi} & 0 \\
0 & D^{n} D^{\pi}
\end{array}\right]\left[\begin{array}{cc}
0 & \left(A^{\pi} C B\right)^{d} A^{\pi} C \\
D^{\pi} B\left(A^{\pi} C B\right)^{d} &
\end{array}\right]^{n+1}\right] \\
& +\sum_{i=0}^{q-1}\left(P_{1}^{\#}\right)^{i+1}\left(I+P_{1}^{\#}\left[\begin{array}{cc}
0 & A^{e} C D^{\pi} \\
D^{e} B A^{\pi} & 0
\end{array}\right]\right)\left[\begin{array}{cc}
A A^{\pi} & A^{\pi} C \\
D^{\pi} B & D D^{\pi}
\end{array}\right]^{i} \\
& \times\left[\begin{array}{cc}
\left(A^{\pi} C B\right)^{\pi} & 0 \\
0 & \left(D^{\pi} B C\right)^{\pi}
\end{array}\right] \\
& \text { - } \sum_{m=1}^{t-1}\left[\begin{array}{cc}
A^{m} A^{\pi} & 0 \\
0 & D^{m} D^{\pi}
\end{array}\right]\left[\begin{array}{cc}
0 & \left(A^{\pi} C B\right)^{d} A^{\pi} C \\
D^{\pi} B\left(A^{\pi} C B\right)^{d} &
\end{array}\right]^{m} \text {, }
\end{aligned}
$$

where $t=\max \{\operatorname{ind}(A), \operatorname{ind}(D)\}, q=\operatorname{ind}\left(\left[\begin{array}{cc}A A^{\pi} & A^{\pi} C \\ D^{\pi} B & D D^{\pi}\end{array}\right]\right)$ and $P_{1}^{\#}$ is defined as in Theorem 4.2

Proof. Using the following decompositions

$$
\begin{gathered}
M=\left[\begin{array}{cc}
A A^{e} & A^{e} C \\
D^{e} B & D D^{e}
\end{array}\right]+\left[\begin{array}{cc}
A A^{\pi} & A^{\pi} C \\
D^{\pi} B & D D^{\pi}
\end{array}\right]:=P+Q, \\
P=\left[\begin{array}{cc}
A A^{e} & A^{e} C D^{e} \\
D^{e} B A^{e} & D D^{e}
\end{array}\right]+\left[\begin{array}{cc}
0 & A^{e} C D^{\pi} \\
D^{e} B A^{\pi} & 0
\end{array}\right]:=P_{1}+P_{2}, \\
Q=\left[\begin{array}{cc}
A A^{\pi} & 0 \\
0 & D D^{\pi}
\end{array}\right]+\left[\begin{array}{cc}
0 & A^{\pi} C \\
D^{\pi} B & 0
\end{array}\right]:=Q_{1}+Q_{2},
\end{gathered}
$$

we establish (4.4) similar to the proof of Theorem 4.2.

Applying Theorem 4.4, we obtain the following result. 
Corollary 4.5. Let $t, q$ and $P_{1}^{\#}$ be defined as in Theorem 4.4

(a) If $C D=0$ and $B A=0$, then

$$
\begin{aligned}
M^{d} & =\left[\begin{array}{cc}
-A^{d} C B\left(A^{\pi} C B\right)^{d} & \left(A^{\pi} C B\right)^{d} A^{\pi} C \\
D^{\pi} B\left(A^{\pi} C B\right)^{d} & -D^{d} B\left(A^{\pi} C B\right)^{d} C
\end{array}\right] \\
& +\sum_{n=1}^{t-1}\left[\begin{array}{cc}
A^{n} A^{\pi} & 0 \\
0 & D^{n} D^{\pi}
\end{array}\right]\left[\begin{array}{cc}
0 & \left(A^{\pi} C B\right)^{d} A^{\pi} C \\
D^{\pi} B\left(A^{\pi} C B\right)^{d}
\end{array}\right]^{n+1} \\
& +\sum_{i=0}^{q-1}\left[\begin{array}{cc}
\left(A^{d}\right)^{i+1} & \left(A^{d}\right)^{i+2} C \\
\left(D^{d}\right)^{i+2} B & \left(D^{d}\right)^{i+1}
\end{array}\right]\left[\begin{array}{ll}
A A^{\pi} & A^{\pi} C \\
D^{\pi} B & D D^{\pi}
\end{array}\right]^{i} \\
& \times\left[\begin{array}{cc}
\left(A^{\pi} C B\right)^{\pi} & 0 \\
0 & \left(D^{\pi} B C\right)^{\pi}
\end{array}\right] \\
& -\sum_{m=1}^{t-1}\left[\begin{array}{cc}
A^{m} A^{\pi} & 0 \\
0 & D^{m} D^{\pi}
\end{array}\right]\left[\begin{array}{cc}
0 & \left(A^{\pi} C B\right)^{d} C \\
D^{\pi} B\left(A^{\pi} C B\right)^{d}
\end{array}\right.
\end{aligned}
$$

(b) If any of (1)-(12) in Theorem 2.2 is valid, $A^{\pi} C=0$, and $D^{\pi} B=0$, then

$$
M^{d}=\sum_{i=0}^{t-1}\left(P_{1}^{\#}\right)^{i+1}\left(I+P_{1}^{\#}\left[\begin{array}{cc}
0 & C D^{\pi} \\
B A^{\pi} & 0
\end{array}\right]\right)\left[\begin{array}{cc}
A A^{\pi} & 0 \\
0 & D D^{\pi}
\end{array}\right]^{i} .
$$

We now provide an example to illustrate our results.

Example 4.6. Consider the $2 \times 2$ block matrices $M=\left[\begin{array}{cc}A & C \\ B & D\end{array}\right]$, where $A=\left[\begin{array}{lll}1 & 1 & 0 \\ 0 & 0 & 0 \\ 0 & 0 & 0\end{array}\right], C=\left[\begin{array}{ll}0 & 0 \\ 0 & 0 \\ 0 & 2\end{array}\right], B=\left[\begin{array}{lll}1 & 1 & 1 \\ 0 & 0 & 0\end{array}\right]$, and $D=\left[\begin{array}{ll}0 & 1 \\ 0 & 0\end{array}\right]$. Then $A^{\#}=A$ and $D^{d}=0$. Since $A C=0$ and $D B=0$, we apply Corollary 4.3 (a) to obtain

$$
M^{d}=\left[\begin{array}{lllll}
1 & 1 & 0 & 0 & 0 \\
0 & 0 & 0 & 0 & 0 \\
0 & 0 & 0 & 0 & 0 \\
1 & 1 & 0 & 0 & 0 \\
0 & 0 & 0 & 0 & 0
\end{array}\right]
$$

\section{REFERENCES}

1. J.K. Baksalary, O.M. Baksalary, G. Trenkler, A revisitation of fomulae for the MoorePenrose inverse of modified matrices, Linear Algebra Appl. 372: 207-224, 2003.

2. A. Ben-Israel, T.N.E. Greville, Generalized Inverses: Theory and Applications, 2nd ed., Springer-Verlag, 2003.

3. S.L. Campbell, C.D. Meyer Jr., N.J. Rose, Applications of the Drazin inverse to linear systems of differential equations, SIAM J. Appl. Math. 31: 411-425, 1976. 
4. S.L. Campbell, C.D. Meyer, Generalized Inverses of Linear Transformations, Dover, New York, 1991.

5. S.L. Campbell, The Drazin inverse and systems of second order linear differential equations, Linear Multilinear Algebra 14: 195-198, 1983.

6. N. Castro-González, Group inverse of modified matrices over an arbitrary ring, Electron. J. Linear Algebra 26: 201-214, 2013.

7. N. Castro-González, J.J. Koliha, Y. Wei, Perturbation of the Drazin inverse for matrices with equal eigenprojections at zero, Linear Algebra Appl. 312: 181-189, 2000.

8. M. Catral, D. D. Olesky, P. Van Den Driessche, Block representations of the Drazin inverse of a bipartite matrix, Electron. J. Linear Algebra 18: 98-107, 2009.

9. J. Chen, J. Xu, Representations for the weighted Drazin inverse of a modified matrix, Appl. Math. Comput. 203: 202-209, 2008.

10. C. Deng, A generalization of the Sherman-Morrison-Woodbury formula, Appl. Math. Lett. 24: 1561-1564, 2011.

11. E. Dopazo, M.F. Martínez-Serrano, On deriving the Drazin inverse of a modified matrix, Linear Algebra Appl. 438: 1678-1687, 2013.

12. W. W. Hager, Updating the inverse of a matrix, SIAM Rev. 31: 221-239, 1989.

13. R.E. Hartwig, X. Li, Y. Wei, Representations for the Drazin inverse of $2 \times 2$ block matrix, SIAM J. Matrix Anal. Appl. 27: 757-771, 2006.

14. R.E. Hartwig, J.M. Shoaf, Group inverses and Drazin inverses of bidiagonal and triangular Toeplitz matrices, J. Austral. Math. Soc. 24: 10-34, 1977.

15. R.E. Hartwig, G. Wang, Y. Wei, Some additive results on Drazin inverse, Linear Algebra Appl. 322: 207-217, 2001.

16. J.J. Koliha, I. Straškraba, Power bounded and exponentially bounded matrices, Appl. Math. 44: 289-308, 1999.

17. T.Y. Lam, Corner ring theory: a generalization of Peirce decompositions, I, Algebras, rings and their representations, World Sci. Publ., Hackensack, NJ, 153-182, 2006.

18. T.Y. Lam, A First Course in Noncommutative Rings, GTM 131, Springer-Verlag, New York, 1991.

19. C.D. Meyer, Generalized inversion of modified matrices, SIAM J. Appl. Math. 24: 315-323, 1973.

20. C.D. Meyer, N.J. Rose, The index and the Drazin inverse of block triangular matrices, SIAM J. Appl. Math. 33: 1-7, 1977.

21. D. Mosić, Some results on the Drazin inverse of a modified matrix, Calcolo 50: 305311, 2013.

22. A. Shakoor, H. Yang, I. Ali, Some representations for the Drazin inverse of a modified matrix, Calcolo 51: 505-514, 2014.

23. J. Sherman, W.J. Morrison, Adjustment of an inverse matrix corresponding to a change in one element of a given matrix, Ann. Math. Statist. 21: 124-127, 1950.

24. Y. Wei, The weighted Moore-Penrose inverse of modified matrices, Appl. Math. Comput. 122: 1-13, 2001.

25. Y. Wei, The Drazin inverse of a modified matrix, Appl. Math. Comput. 125: 295-301, 2002.

26. M.A. Woodbury, Inverting Modified Matrices, Technical Report 42, Statistical Research Group, Princeton University, Princeton, NJ, 1950.

27. D. Zhang, X. Du, Representations for the Drazin Inverse of a Modified Matrix, Filomat 29: 853-863, 2015. 
College of Sciences, Northeast Electric Power University, Jilin, P.R. China.

E-mail address: daochangzhang@126.com

Faculty of Sciences and Mathematics, University of Niš, P.O. Box 224, 18000 Niš, Serbia.

E-mail address: dijana@pmf.ni.ac.rs

Department of Mathematics and Statistics, University of Nevada, Reno, NV 89557, USA.

E-mail address: ttam@unr.edu 